\title{
The Role of Immunosuppression in the Transplantation of Allogenic Neural Precursors Derived from Human Pluripotent Stem Cells for Parkinson's Disease
}

\author{
Casimir de Rham ${ }^{1}$, Vannary Tieng², Benjamin B Tournier ${ }^{3}$, Yannick Avila ${ }^{1}$, Nathalie Ginovart ${ }^{3}$, Karl-Heinz Krause ${ }^{2}$, Olivier Preynat-Seauve ${ }^{2}$ \\ Michel Dubois-Dauphin ${ }^{2}$ and Jean Villard ${ }^{1 \star}$
}

${ }^{1}$ Transplant Immunology Unit, Division of Immunology and Allergy, Division of Laboratory medicine, Department of Medicine Geneva, Switzerland ${ }^{2}$ Department of Pathology and Immunology, Faculty of Medicine, University of Geneva, Switzerland

${ }^{3}$ Department of Psychiatry, Faculty of Medicine, University of Geneva, Switzerland

\begin{abstract}
Objective: Neural progenitor cells (NPC) derived from human embryonic stem cells have the potential to differentiate into mature neurons after transplantation in the brain, opening the possibility of regenerative cell therapy for neurodegenerative disorders like Parkinson's disease. For such therapy, the source of NPC is genetically unrelated to the patient, leading to potential rejection of the transplanted cells by the host's immune response. Rejection can be prevented by the use of immunosuppressive drugs (ISD). Previous works have suggested that cyclosporine and dexamethasone used in classical immunosuppressive regimen could prevent the terminal differentiation of NPC into mature neurons depending on culture conditions.
\end{abstract}

Methods: We have investigated in vitro the role of other ISD, Intra venous Immunoglobulins (IVIG), mycophenolate mofetil and tacrolimus. We have tested the immunosuppressive activity of tacrolimus and cyclosporine on the effector of natural killer (NK) and $\mathrm{CD}^{+} \mathrm{T}$-cells and performed a microarray to analyse the difference between the two drugs for the neuron differentiation. Finally, human transplanted neuroprecursor cell survival has been analyzed in rats treated with tacrolimus or cyclosporine and anti-inflammatory treatments.

Results: IVIG and mycophenolate mofetil interfere with the development of NPC into mature neurons, but tacrolimus do not inhibit the maturation process of NPC. Microarray experiments demonstrate significant differences between cyclosporine and tacrolimus gene expression during NPC maturation into mature neurons. Tacrolimus like cyclosporine is able to inhibit the $\mathrm{CD} 8^{+} \mathrm{T}$-cells activation against neural progenitors, but both are unable to block NK cells activity. NK cells could be potential harmful weapons to reject NPC and mature neurons. In rats treated with both immunosuppressive (tacrolimus or cyclosporine) and anti-inflammatory treatments, engrafted human neuroprecursors cell survival is good and the microglial density is low.

Conclusion: These data suggest in vivo that both tacrolimus and cyclosporine, with an anti-inflammatory treatment like prednisolone, promote graft survival and minimise the host microglial response.

Keywords: Human pluripotent stem cells; Neuron precursor cells; NK cells; T cells; Tacrolimus; Cyclosporine; Prednisolone; Immunosuppression; Parkinson's disease

Abbreviations: NPC: Neural Precursor Cells; ISD: Immunosuppressive Drugs; IvIg: Intravenous Immuneglobulins; NK: Natural Killer Cells; hES: Human Embryonic Stem Cells; MMF: Mycophenolate Mofetil; CsA: Cylcosporine A; TaC: Tacrolimus; NFAT: Nuclear Factor of Activated T-cells; FKBP: FK Binding Protein; HCM: Human Cytoplasmic Marker; MHC: Major Histocompatibility Complex; hiPS: Human Induced Pluripotent Stem Cells

\section{Introduction}

Immense expectations surround the field of stem-cells transplantation. However, considering the potential risk of rejection by the recipient's immune system, the source of stem cells has to be carefully evaluated. Due to their various characteristics, human embryonic stem (hES) cells provide an excellent tool for transplantation. The most important attribute of hES cells is their pluripotency, since they can differentiate into the three different germ layers, i.e. endoderm, mesoderm and ectoderm, and they can be effective for all types of differentiated cells. Another advantage is their capacity to indefinitely proliferate in the appropriate culture conditions. Investigations carried out on hES cells have always been surrounded by controversy, and for many, the use of embryos, to produce cell lines, still presents an ethical dilemma. In addition, even though the potential of hES cells is huge, there are still many hurdles ahead before the clinical use of these cells.

The origin of pluripotent cells is of great importance. Indeed, in an allogenic transplantation model, in which the donor is genetically unrelated to the patient, an immune response will be triggered by the recipient.

Seminal publications strongly suggest that pluripotent cells, such as $\mathrm{hES}$ cells possess immune-privileged properties [1-3].

*Corresponding author: Jean Villard, MD, PhD, Transplant Immunology Unit, Geneva University Hospital, 4, rue Gabrielle Perret-Gentil, 1211 Geneva 14, Switzerland, Tel:+4122 3729394/+4179 5533409; Fax: +41 22 3729390; E-mail: Jean.Villard@hcuge.ch

Received November 21, 2013; Accepted December 18, 2013; Published December 20, 2013

Citation: de Rham C, Tieng V, Tournier BB, Avila Y, Ginovart N, et al. (2013) The Role of Immunosuppression in the Transplantation of Allogenic Neural Precursors Derived from Human Pluripotent Stem Cells for Parkinson's Disease. J Stem Cell Res Ther S6: 006. doi:10.4172/2157-7633.S6-006

Copyright: (C) 2013 Rham C, et al. This is an open-access article distributed unde the terms of the Creative Commons Attribution License, which permits unrestricted use, distribution, and reproduction in any medium, provided the original author and source are credited. 
Citation: de Rham C, Tieng V, Tournier BB, Avila Y, Ginovart N, et al. (2013) The Role of Immunosuppression in the Transplantation of Allogenic Neural Precursors Derived from Human Pluripotent Stem Cells for Parkinson's Disease. J Stem Cell Res Ther S6: 006. doi:10.4172/21577633.S6-006

Page 2 of 9

Thus, transplantation of hES into organs like the brain, which is also considered as an immune-privileged site, was viewed as a typical example of a potentially curative treatment of neurodegenerative diseases without occuring the risk of an unwanted immune reaction. More recent works imply that the risk of rejection by the immune system of allogenic hES cells, or their progenitors, warrants serious thoughts. $\mathrm{T}$ lymphocytes and natural killer (NK) cells are able to target $\mathrm{hES}$ cells and their progenitors. That is why immunosuppressive drugs (ISD) have to be included in the transplant protocol. By analogy with organ or tissue transplantation from a genetically unrelated individual, the use of different ISD, which possess diverse mode of action, is recommended to prevent rejection. Thanks to these drugs, graft survival is steadily on the increase.

The aim of ISD is to impede four main cellular mechanisms and to achieve the following effects; (1) Blockade of T-cell proliferation using anti-metabolites like azathioprine or mycophenolate mofetil (MMF); (2) Reduction in inflammation by using steroids; (3) Inhibition of cytokine production by blocking calcineurin with cyclosporine (CsA) or tacrolimus (TaC); (4) Finally, depletion of macrophages/APC, T-cells and/or B-cells with monoclonal antibodies, such as rATG, Rituximab or alemtuzumab.

Steroids or corticosteroids act as glucocorticoid receptor agonists, which target the transcription factors NF- $\mathrm{kB}$. This therapy is used to reduce inflammation and to induce T-cell apoptosis. Steroids are still very important immunosuppressors, especially in the first month after transplantation. Azathioprine was the first immunosuppressive drug to be used on a regular basis in organ transplantation. It acts by releasing a compound, 6-mercaptopurine, which blocks DNA synthesis. Azathioprine has been replaced progressively by mycophenolate mofetil, which inhibits inosine-monophosphate dehydrogenase, an enzyme involved in the synthesis of purines.

CsA and $\mathrm{TaC}$ are two compounds that interact with the calcineurin protein. Calcineurin binds to a transcription factor, nuclear factor of activated T-cells (NFAT), and this complex increases the expression of IL-2 and stimulates the development and the differentiation of T-cells. Depending on patients and side effects, either CsA or $\mathrm{TaC}$ is used, but never both together.

In a previous work, we have demonstrated that CsA and high doses of dexamethasone could inhibit the last step of neuron differentiation from precursors in vitro; in addition, CsA was unable to inhibit the cytotoxicity of NK cells. Therefore, the best immunosuppressive regimen should be able to block NK and T-cells without inhibiting the differentiation of precursors into mature cells.

In this new work, we analyzed additional ISD during the last step of neuron differentiation first in vitro. Then, we tried to optimize in rats transplanted with NPC, the best immunosuppressive regimen to prevent inflammation and rejection.

\section{Material and Methods}

\section{hES cell culture}

H1 hES cell line was acquired from WiCell Research Institute (Madison, WI, USA) at low passage under the authorization of the Swiss Public Health Service (OFSP N R-FP-S-2-0004-00-4). Cells were expanded under non-differentiating conditions and analyzed between passages 10 and 50 . They were maintained as recommended by the supplier in human ES cell medium consisting of 80\% DMEM/F12, 20\% KnockOut-Serum Replacement, 2 mM L-glutamine, 1\% non-essential amino acids, $0.1 \mathrm{mM} \beta$-mercaptoethanol, $4 \mathrm{ng} / \mathrm{ml}$ basic Fibroblast Growth Factor (bFGF) (Invitrogen, Carlsbad, CA, USA) at $37^{\circ} \mathrm{C}$, $5 \% \mathrm{CO}_{2}$ and high humidity. Mouse embryonic fibroblasts, isolated from embryos of pregnant CF-1 mice (Charles River Laboratories, Wilmington, MA, USA), were used as feeder cells. Fibroblasts were cultured until passage 2 in DMEM (Invitrogen, Carlsbad, CA, USA) supplemented with $10 \%$ foetal bovine serum and $1 \%$ penicillin/ streptomycin (Invitrogen, Carlsbad, CA, USA). Cells were mitotically inactivated by irradiation at 40 Gy before seeding on a gelatin-coated 6-well plate at $1.9 \times 10^{5}$ cells/plate. hES cell culture medium was changed daily, and the cells were plated every 8 days following incubation with type IV collagenase $\left(1 \mathrm{mg} / \mathrm{ml}\right.$; Invitrogen) for $20-30 \mathrm{~min}$ at $37^{\circ} \mathrm{C}$ and mild mechanical disruption.

\section{Neural differentiation}

For differentiation of HESC towards NPC, HESC were dissociated with type IV collagenase $(1 \mathrm{mg} / \mathrm{ml})$ and colonies were maintained in suspension for one week in neural induction medium (DMEMF-12, penicillin/streptomycin 1\%, N-2 supplement (Gibco, Invitrogen, Carlsbad, CA, USA ). NPC-containing aggregates were then plated at low density (10-20 aggregates) onto 6-well plate coated with laminin and cultured in neural induction medium for two additional weeks in the presence of $100 \mathrm{ng} / \mathrm{ml} \mathrm{bFGF}$ and $100 \mathrm{ng} / \mathrm{ml} \mathrm{EGF} \mathrm{(R \& D} \mathrm{Systems}$ Inc., Minneapolis, MN, USA).

Neuronal differentiation of NPC was achieved by cell dissociation of 3 week aggregates with $0.5 \%$ trypsin/EDTA (Gibco, Invitrogen, Carlsbad, CA, USA) and re-plated at low density $\left(5000 \mathrm{cells} / \mathrm{cm}^{2}\right)$ on 1 $\mu \mathrm{g} / \mathrm{ml}$-laminin-coated dishes in Neurobasal medium (Gibco, Invitrogen, Carlsbad, CA) supplemented with B-27 supplement (Gibco, Invitrogen, Carlsbad, CA, USA), BDNF 10 ng/ml (R\&D Systems Inc., Minneapolis, $\mathrm{MN}$, USA), $1 \%$ penicillin/streptomycin. Immunosuppressive drugs (Tacrolimus, mycophenolate mofetil, IVIg and cyclosporine) were added or not in medium at the beginning of neuronal differentiation.

\section{NK cells and $\mathrm{CD8}^{+} \mathrm{T}$-cells isolation}

Peripheral blood mononuclear cells (PBMC) were isolated from normal blood donors by density-gradient centrifugation. NK cells were separated from PBMC by magnetic cell sorting according to the manufacturer's instructions (NK cell isolation kit, Miltenyibiotec, Bergisch Gladbach, Germany). Non-NK cells from human PBMC, such as $\mathrm{T}$ cells, B cells, dendritic cells, monocytes, granulocytes and erythrocytes were stained with a cocktail of biotin-conjugated antibodies to CD3, CD4, CD14, CD15, CD19, CD36, CD123 and CD123a. A second staining was made using an anti-biotin monoclonal antibody $(\mathrm{mAb})$ conjugated with microbeads. The NK cells were isolated by depletion of the magnetically labeled cells. The isolated NK cells were cultured for 5 days in RPMI medium supplemented with $10 \%$ human $\mathrm{AB}$ serum, $100 \mathrm{U} / \mathrm{ml}$ penicillin, $100 \mu \mathrm{g} / \mathrm{ml}$ streptomycin, $2 \mathrm{mM}$ L-glutamine, 1\% MEM non-essential amino acids and $0.1 \mathrm{mM}$ sodium pyruvate, $5 \mathrm{mM} \beta$-ME (at $5 \times 10^{-5} \mathrm{M}$ ). In some experiments, 25 $\mathrm{ng} / \mathrm{ml}$ of recombinant human IL-15 (R\&D Systems Inc., Minneapolis, MN, USA) were added. Cyclosporine A (Novartis AG), Tacrolimus (Astellas AG) and Mycophenolate Mofetil (Roche) and intravenous polyclonal Ig (CSL Behring) were added to the culture at different concentration. $\mathrm{CD}^{+} \mathrm{T}$-cells were separated from PBMC by magnetic cell sorting according to the manufacturer's instructions $\left(\mathrm{CD}^{+} \mathrm{T}\right.$-cells isolation kit, Miltenyibiotec, Bergisch Gladbach, Germany). Non$\mathrm{CD}^{+}$T-cells from human PBMC, such as $\mathrm{CD} 4^{+} \mathrm{T}$-cells, B-cells, NK cells, monocytes, neutrophiles dentritic cells, etc. were stained with a cocktail of biotin-conjugated antibodies to CD4, CD15, CD16, CD19, 
Citation: de Rham C, Tieng V, Tournier BB, Avila Y, Ginovart N, et al. (2013) The Role of Immunosuppression in the Transplantation of Allogenic Neural Precursors Derived from Human Pluripotent Stem Cells for Parkinson's Disease. J Stem Cell Res Ther S6: 006. doi:10.4172/21577633.S6-006

Page 3 of 9

CD34, CD36, CD56, CD123, TCR $\gamma / \delta$ AND CD235a. A second staining was made using an anti-biotin monoclonal antibody $(\mathrm{mAb})$ conjugated with microbeads. The $\mathrm{CD} 8^{+} \mathrm{T}$-cells were isolated by depletion of the magnetically labeled cells. The isolated $\mathrm{CD}^{+} \mathrm{T}$-cells were cultured for 5 days in RPMI medium supplemented with $10 \%$ FCS serum, $100 \mathrm{U} /$ $\mathrm{ml}$ penicillin, $100 \mu \mathrm{g} / \mathrm{ml}$ streptomycin, $2 \mathrm{mM}$ L-glutamine, $1 \% \mathrm{MEM}$ non-essential amino acids and $0.1 \mathrm{mM}$ sodium pyruvate, $5 \mathrm{mM} \beta$-ME (at $5 \times 10^{-5} \mathrm{M}$ ). In some experiments, $1000 \mathrm{U} / \mathrm{ml}$ of recombinant human IL-2 (Bio-gen Inc. Cambridge, MA, USA) Cyclosporine A (Novartis AG), Tacrolimus (Astellas AG), Mycophenolate Mofetil (Roche) and intravenous polyclonal Ig (CSL Behring) were added to the culture at different concentrations.

\section{Antibodies}

The following antibodies were used to characterize NPC and neurons by immunostaining: rabbit anti-nestin (Chemicon, Millipore, San Francisco, CA, USA), rabbit anti- $\beta$ III-tubulin (Covance, Princeton, NJ, USA). The following mouse anti-human antibodies were purchased from BD PharMingen ${ }^{\text {TM }}$ (San Jose, CA, USA): PE-Cy7-conjugated antiCD3, APC-Cy7-conjugated anti-CD16, FITC-conjugated anti-CD107a and PE-conjugated anti-HLA-I. APC-conjugated anti-CD56 was from Miltenyibiotec (Bergisch Gladbach, Germany).

\section{Set up of a non-radioactive assay with flow cytometry analysis}

We set up an in vitro model that allowed us analyzing simultaneously the killing of allogenic NPC and the phenotype or the functionality of the involved NK/ CD8 ${ }^{+}$T-cells.

K562 and allogenic NPC were labelled with Vybrant ${ }^{\circledR}$ Multicolor Cell-Labelling Kit (DiO solution, Invitrogen; Eugene, OR, USA). DiO solution was dissolved in $100 \%$ ethanol (1/10 dilution) and added to the cell suspension for $10 \mathrm{~min}$ at $37^{\circ} \mathrm{C}$. Cells were washed 3 times with PBS $+0.5 \%$ FCS and resuspended in cell media. Then, previously incubated $\mathrm{NK} / \mathrm{CD}^{+}$T-cells isolated from healthy donors were cocultured with the different target cells, at a ratio of $1: 1$, for 4 hours in $5 \%$ $\mathrm{CO}_{2}$-air humidified atmosphere at $37^{\circ} \mathrm{C} .7-\mathrm{AAD}$ was added at $10 \mu \mathrm{g} /$ $\mathrm{ml}$ final concentration to the harvested cells $\left(\mathrm{NK} / \mathrm{CD} 8^{+} \mathrm{T}\right.$ - and target cells). Flow cytometry experiments were performed using FACS-CyAn and Summit software (Beckmann Coulter, USA).

\section{Immunofluorescence analysis}

Cells for analysis were cultured and stimulated on glass cover slips before immunofluorescent staining. Samples were fixed in PBS- $4 \%$ paraformaldehyde for 30 minutes at room temperature and permeabilized for half an hour with PBS containing $0.2 \%$ triton X-100. Cells were then incubated overnight at $+4^{\circ} \mathrm{C}$ with primary antibodies in PBS containing $1 \%$ fetal bovine serum. After washing in PBS, secondary antibodies were added for $1 \mathrm{~h} \mathrm{30}$ ' at room temperature with the same buffer and cell nuclei were stained with DAPI $300 \mathrm{nM}$ (in PBS) for 15 additional minutes. Sections were finally washed twice in PBS, rinsed with water before mounting in FluorSave ${ }^{\circledR}$ medium (Calbiochem, SanDiego, CA, USA).

\section{CD107a expression}

For CD107a expression, NK cells were briefly incubated with $\mathrm{K} 562$ or NPC. After $1 \mathrm{~h}$ at $37^{\circ} \mathrm{C}$ in $5 \% \mathrm{CO}_{2}$ air humidified atmosphere, $\mathrm{CD} 107 \mathrm{a}$ and monensin $(6 \mathrm{ug} / \mathrm{ml})$ were added for $5 \mathrm{~h}$ and CD107a expression was measured by flow cytometry.

\section{Microarrays gene expression}

Total RNA was isolated and quality controlled for RNA integrity by capillary electrophoresis on Agilent 2100 Bioanalyzer. $400 \mathrm{ng}$ were amplified and labeled using the Illumina TotalPrep RNA Amplification kit (Ambion). The cRNA quality was assessed by capillary electrophoresis on Agilent 2100 Bioanalyzer. Hybridization on HumanHT-12 v4 expression arrays (Illumina) was carried out according to the manufacturer's instructions.

Data were normalized and analyzed using Illumina GenomeStudio software (background correction and quantile normalization). To assess the difference in gene-expression values between NPC and $\mathrm{NPC}+$ Tacrolimus (NPC+Tacro) or NPC+Cyclosporine (NPC+Cyclo), we did a 1-way ANOVA with contrast in Partek Genomics Suite (http:// www.partek.com). P-values were corrected for multiple testing by use of the false-discovery rate (FDR) method of Benjamini and Hochberg. We applied a conservative significance threshold of 5\% FDR associated with fold change value of 1.5 or more. Pathway analysis of the genes, which were identified as differentially expressed by microarray experiment, was undertaken by using the MetaCore software (http:// www.genego.com).

The gene expression data can be found in ArrayExpress (http:// www.ebi.ac.uk/microarray-as/ae).

\section{Animal experiments}

Eight male Sprague Dawley rats weighing 275-300 g were anaesthetized with $1.5 \%$ isoflurane. Using a $5 \mu \mathrm{l}$ Hamilton microsyringe with a 26 gauge needle, a total of 200,000 cells in $2 \mu$ l were transplanted into the left striatum at the following stereotaxic coordinates: AP: +0.07 $\mathrm{mm}$ from Bregma, ML: $+0.25 \mathrm{~mm}$ from Bregma, $\mathrm{V}:-0.45$ and $-0.55 \mathrm{~mm}$ from the dura; incisor bar set $-3.3 \mathrm{~mm}$ below the interaural line. Half of the cell suspension was infused over 3-min at each of the two sites, with a 3-min waiting period between the first and second deposits. Before retraction, the syringe was left in place for 3-min to minimize cell reflux along the needle tract. After surgery, all animals received buprenorphine $\left(0.02 \mathrm{mg} / \mathrm{kg}\right.$; sc; Temgesic ${ }^{\mathbb{R}}$, Reckitt Benckiser Pharmaceuticals Inc.) for post-operative analgesia. The rats were randomized to 1 of 4 treatment regimens. The control group received 12 daily i.p. injections of saline starting 2 days prior to grafting, followed by 5 daily i.p. injections per week until perfusion 1 month after grafting. The tacrolimus group received 12 daily i.p. injections of tacrolimus ( $1.5 \mathrm{mg} / \mathrm{kg}$; FK506, Toku) starting 2 days prior to grafting, followed by 5 daily i.p. injections per week until perfusion. The tacrolimus+prednisolone group received the tacrolimus treatment for one month (as above) and daily i.p. prednisolone $\left(20 \mathrm{mg} / \mathrm{kg}\right.$; Solu-medrol ${ }^{\circledR}$, Pfizer) for 9 days after grafting. The cyclosporine+prednisolone group received 12 daily i.p. injections of cyclosporine A (Sandimmun Neoral ${ }^{\circledR}$, Novartis) starting 2 days prior to grafting (20 and $15 \mathrm{mg} / \mathrm{kg}$ for the first six and last six injections, respectively). Cyclosporine A was then administered as 5 daily i.p. injections from Mondays to Fridays $(15 \mathrm{mg} / \mathrm{kg}$ ) and as $100 \mu \mathrm{g} / \mathrm{ml}$ in the drinking water during the weekends, until perfusion. Rats in this group also received daily i.p. injections of prednisolone $(20 \mathrm{mg} / \mathrm{kg})$ for the first 9 days following grafting.

Experiments were performed in accordance with the Swiss Federal Law on animal care and were approved by the Swiss Cantonal Veterinary Office (protocol 1016/3490/2).

\section{Phenotypic analysis of injected surviving cells}

At one month after grafting, rats underwent intracardiac perfusion with $4 \%$ paraformaldehyde, their brain was removed and embedded in paraffin. Ten- $\mu \mathrm{m}$ thick coronal sections were obtained using a microtome. Double immunohistochemical detection was performed 
Citation: de Rham C, Tieng V, Tournier BB, Avila Y, Ginovart N, et al. (2013) The Role of Immunosuppression in the Transplantation of Allogenic Neural Precursors Derived from Human Pluripotent Stem Cells for Parkinson's Disease. J Stem Cell Res Ther S6: 006. doi:10.4172/21577633.S6-006

Page 4 of 9

with the primary antibodies against human cytoplasmic marker (HCM; 1/500, Stem Cells) and ionized calcium-binding adapter molecule 1 (Iba1; 1/500, microglia, Wako). Detection was performed using Alexa 488- and Alexa 555-labeled secondary antibodies (Invitrogen). Sections were counterstained with 4,6-diamidino-2-phenylindole (DAPI; Sigma-Aldrich). Imaging was performed with a conventional fluorescence microscope (Leitz Axioskop 2 Plus).

\section{Drug preparation}

Tacrolimus (FK506, Toku) was initially dissolved in $80 \%$ ethanol $20 \%$ ricini oleum virginum (Hanseler AG). Tacrolimus $(5 \mathrm{mg} / \mathrm{ml})$, cyclosporine $(100 \mathrm{mg} / \mathrm{ml}$, Sandimmun, Neoral, Novartis) and methylprednisolone $(64.1 \mathrm{mg} / \mathrm{ml}$, Solu-medrol, Pfizer) were diluted in saline solution immediately before injection. All drugs were injected intraperitoneally.

\section{Results}

Effect of different ISD in neural development and differentiation from neural progenitor cells (NPC)

Transplantation of NPC derived from hES cells would lead to rejection by the immune system due genetic differences with the recipient. To prevent rejection, the administration of ISD is mandatory (Figure 1). We have already demonstrated that cyclosporine, a calcineurin inhibitor that is classically used to prevent rejection, inhibits the last stage of neuronal differentiation in vitro. Therefore we investigated other drugs, used to prevent rejection in clinical protocol of solid organ or tissue transplantation. The antimetabolite mycophenolate mofetil that inhibits selectively and reversibly the enzyme inosine monophosphate deshydrogenase, is mainly used by T- and B-cells. The differentiation of neurons from precursors was inhibited by the presence of mycophenolate mofetil at different concentration (Figure $2 \mathrm{~A}+$ Supplementary Figure 1A). Then, we tested tacrolimus, another calcineurin inhibitor. Like cyclosporine, tacrolimus interacts with calcineurin, which binds to the transcription factor NFAT (nuclear factor of activated T-cells). This complex increases the expression of IL-2 and stimulates the development and the differentiation of T-cells.

In contrast to cyclosporine which interacts first with cyclophilin,

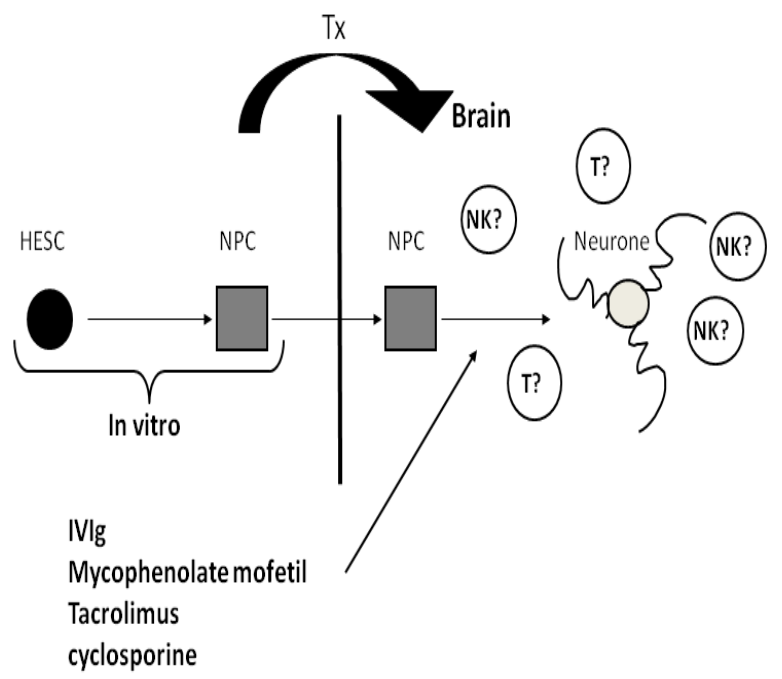

Figure 1: Schematic view of the experiments with immunosuppressive drugs. tacrolimus binds to the FK binding protein (FKBP) to block NFAT. However, compared to cyclosporine and mycophenolate mofetil, tacrolimus did not interfere with the differentiation of NPC into mature neurons (Figure 2B+Supplementary Figure 1B).

Finally, we have tested the IVIg (intra venous polyclonal immune globulin), a mixture of several thousands of plasmas from healthy blood donors and that therefore includes a large repertoire of different immunoglobulin. The main indication of IVIg is the substitution of humoral immunodeficiency (primary or secondary). But administration of IVIg was also indicated to treat autoimmune diseases due to immunomodulatory properties, and in solid organ transplantation, IVIg is used to treat rejection and to reduce the level of anti-HLA antidonor antibodies [4,5]. As expected, due to the large panel of antibodies, the presence of IVIg inhibits the differentiation of NPC into neurons (Figure 2C+Supplementary Figure 1C).

In summary, according to the method of culture used in these experiments, only tacrolimus did not interfere with the differentiation of NPC into mature neurons (Figure 2D), in terms of neuron numbers and neuron clusters.

\section{Microarray analysis of gene expression during the differentiation of neuron from NPC in presence of CsA or $\mathrm{TaC}$}

The difference between the two types of calcineurin inhibitors, cyclosporine and tacrolimus, regarding to the differentiation of NPC into mature neurons was intriguing. To try to understand this observation, we have realized and analyzed a microarray that includes $45^{\prime} 000$ probes to compare NPC cultured in presence of cyclosporine, tacrolimus or in absence of these 2 drugs. NPC were harvested and RNA was extracted and reverse transcripted into cDNA. With this material, a microarray was done to investigate which genes were amplified or induced and diminished or repressed. The threshold was set at 2 (positive and negative) and the P-value was set at 0.05 . With these values, we found that 78 genes modulate their expression in the presence of cyclosporine, whereas 132 genes were altered with tacrolimus. All these genes play different role in different biological processes. But some of them, which are amplified or induced as well as diminished or repressed, are involved in the neuronal process. One of these genes, CRYAA, which contributes to the transparency index of the lense is repressed in presence of cyclosporine, and slightly diminished its expression in presence of tacrolimus. Another gene, TCBA1, which is expressed in foetal brain, is also repressed in presence of cyclosporine (data not shown). Then we focused our analysis on the difference between the culture conditions of NPC without ISD or with $\mathrm{TaC}$ compared to NPC cultured in presence of cyclosporine (Figures $3 \mathrm{~A}$ and $3 \mathrm{~B}$ ). Thirty genes were dowregulated or upregulated by a factor 1.5 at least. The most interesting observation was the downregulation of the APOA-IV ( $<6$ fold decrease gene expression).

\section{Killing of NPC by $\mathrm{NK}$ and $\mathrm{CD8}^{+} \mathrm{T}$-cells in presence of cyclosporine or tacrolimus}

The classical chromium release assay is a usual test to determine the capacity of the effector cells to kill target cells. It is a simple test but with several inconvenients. This test uses radioactive chromium $\left({ }^{51} \mathrm{Cr}\right)$, which means that several precautions must be taken. On the other hand, nothing is known about the phenotype and functionality by the effector cells during this assay. That is why a new test, using flow cytometry (FACS), was set up. In one test, the phenotype and the functionality 
Citation: de Rham C, Tieng V, Tournier BB, Avila Y, Ginovart N, et al. (2013) The Role of Immunosuppression in the Transplantation of Allogenic Neural Precursors Derived from Human Pluripotent Stem Cells for Parkinson's Disease. J Stem Cell Res Ther S6: 006. doi:10.4172/21577633.S6-006

\section{A. Mycophenolate Mofetil}
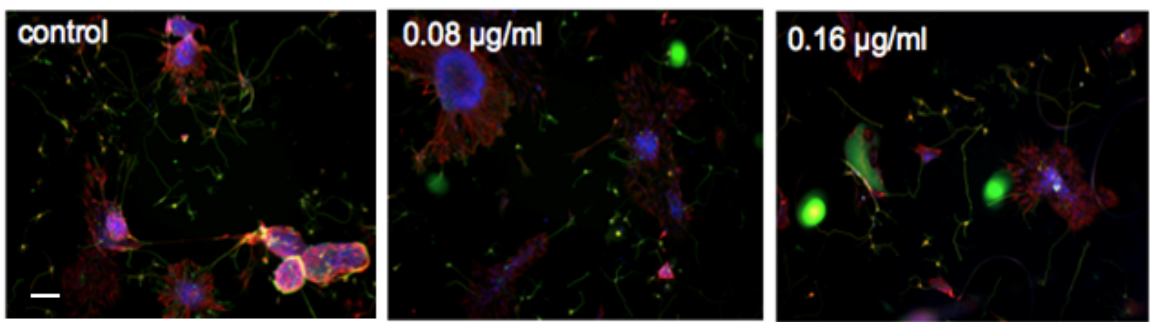

\section{B. Tacrolimus}

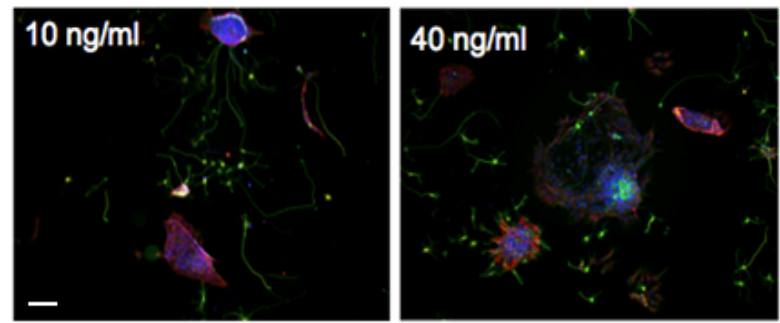

\section{Polyclonal Immunoglobulines (IvIG)}
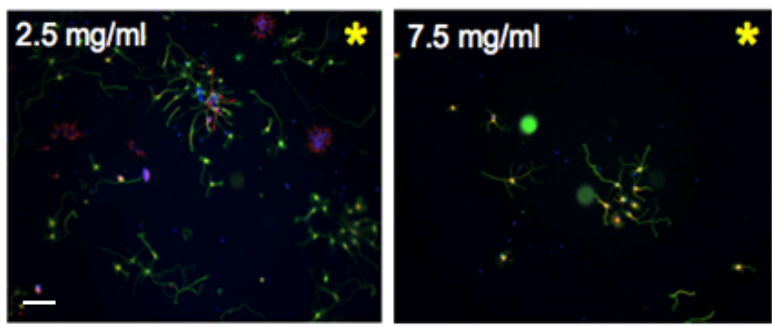

\begin{tabular}{|c|c|c|}
\hline & $\begin{array}{l}\text { Neural } \\
\text { clusters }\end{array}$ & Neurons \\
\hline Ciclosporine & $\downarrow$ & $\downarrow$ \\
\hline Dexamethasone & $\downarrow$ & $\downarrow$ \\
\hline ycophenolate mofetil & $\downarrow$ & $\downarrow$ \\
\hline Vlg immunoglobulins & $\downarrow \downarrow$ & $\downarrow \downarrow$ \\
\hline Tacrolimus & unchanged & unchanged \\
\hline
\end{tabular}

Figure 2: Differentiation of neuron progenitors in presence of different immunosuppressives drugs. A: Control (without immunosuppressive drugs) and Mycophenolate at 2 concentrations.B: Tacrolimus at 2 concentrations. C: Polyclonal immunoglobulines at 2 concentrations. D: Summary of the different immunosuppressive drugs on neuron differentiation and neural cluster formation. Magnification: 10x; scale bar: 100 um.

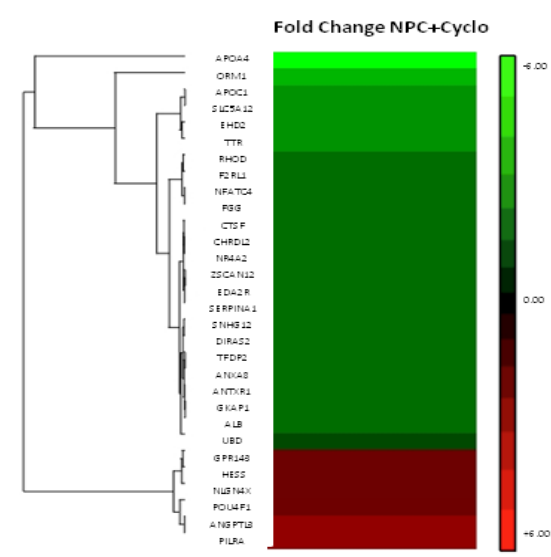

B.

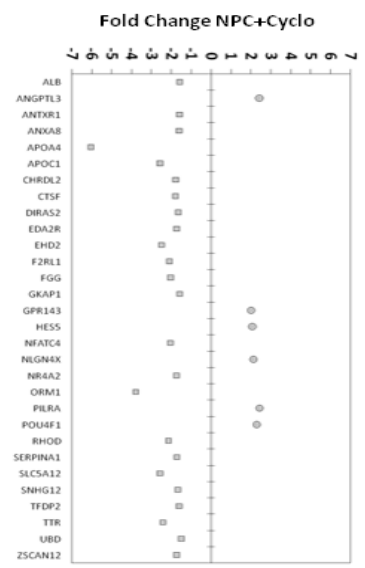

: Fold Change of (NPC+Cyclo/NPC medium orTacro) $\geq 1.5$

$\square:$ Fold Change of (NPC+Cyclo/NPC medium or Tacro) $\leq 1.5$

Figure 3: NPC differentiation co-cultured at early stage with tacrolimus or cyclosporine. A: Heat Map of the co-cultured with medium or tacrolimus compared to cyclosporine. B: Effect of cyclosporine on inhibition of NPC differentiation compared to tacrolimus or to medium and on expression of genes which are downregulated or upregulated by a factor of 1.5 at least. 
Citation: de Rham C, Tieng V, Tournier BB, Avila Y, Ginovart N, et al. (2013) The Role of Immunosuppression in the Transplantation of Allogenic Neural Precursors Derived from Human Pluripotent Stem Cells for Parkinson's Disease. J Stem Cell Res Ther S6: 006. doi:10.4172/21577633.S6-006

Page 6 of 9

(cytotoxicity, CD107a expression and IFN- $\gamma$ secretion) of the effector cells can be analyzed. Briefly, target cells are stained with CFSE; this allows distinguishing them from the effector cells. After the cytotoxic assay, all the cells are harvested and 7-AAD (marker for dead cells) is added just before FACS analysis. Thus the double positive $\mathrm{CFSE}^{+} / 7-$ $\mathrm{AAD}^{+}$population represents the target cells, killed by the effector cells. On one side, target cells can be analyzed by gating on the $\mathrm{CFSE}^{+} / 7$ $\mathrm{AAD}^{+}$population, and on the other side, by gating on the effector cells, therefore their phenotype and functionality can be studied (Figures $4 \mathrm{~A}$ and $4 \mathrm{~B}$ ). This protocol was set up with NK and T-cells as effector cells, cultured with or without cyclosporine or tacrolimus. Cyclosporine and tacrolimus were able to inhibit the killing of NPC by $\mathrm{CD}^{+} \mathrm{T}-$ cells but not the killing by NK cells (Figures $5 \mathrm{~A}$ and $5 \mathrm{~B}$ respectively). When we analyzed the expression of CD107a, a marker of NK and T-cells degranulation, cyclosporine and tacrolimus were able to inhibit
A.

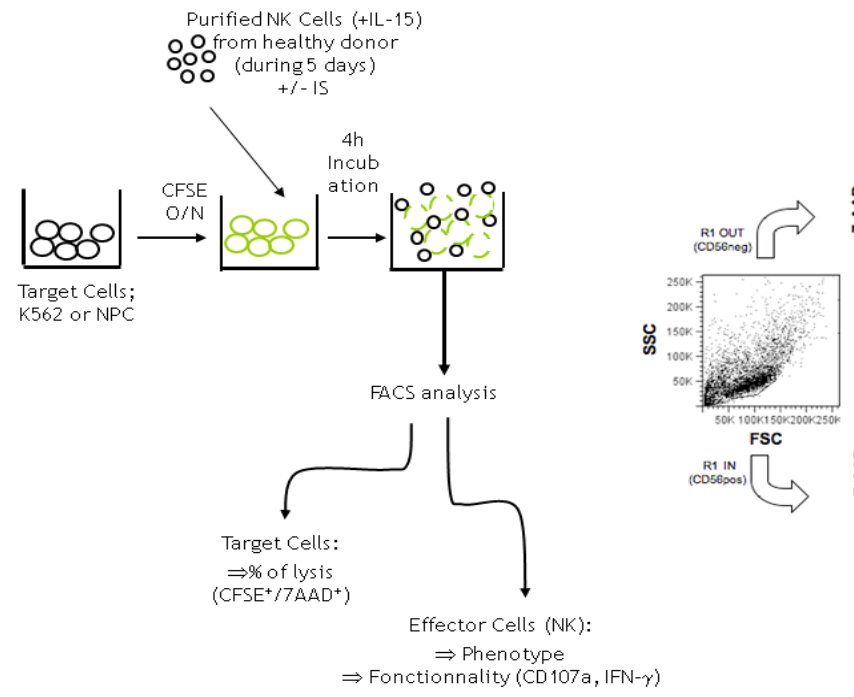

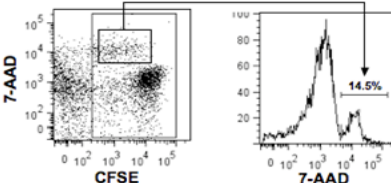
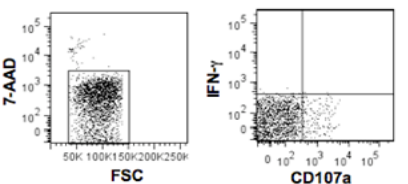

Figure 4: Cytotoxic assay by FACS analysis. A: Schematic view of the cytotoxic assay which allows analysing simultaneously target cells and cytotoxic T or NK cells. B: Prototypical example of cytotoxic assay by FACS with NK cells. NK cells are gated and excluded from the analysis (upper panel), the double positive CFSE+/7AAD+ NPC population represents NPC lysed by the NK cells. When the NK cells are gated on the living cells (lower panel), the expression of CD107a and the secretion of IFN- $\gamma$ can be analysed.

A.

\section{T cells}

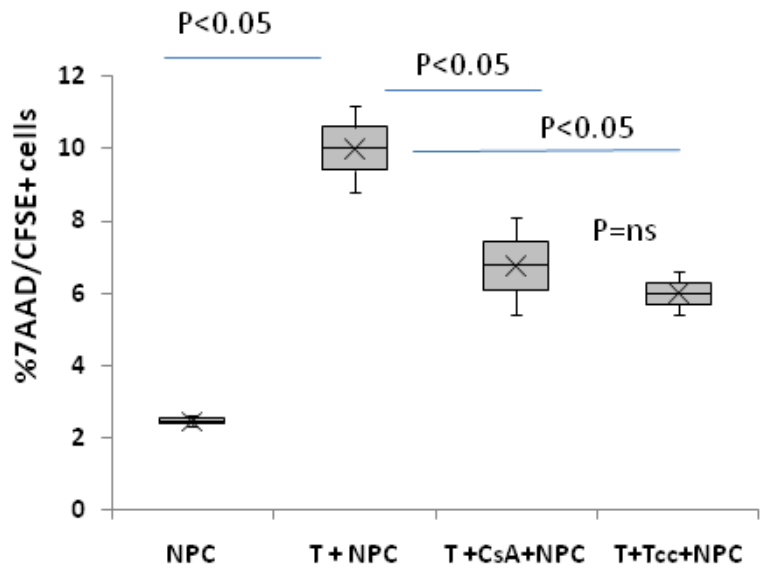

B.

\section{NK cells}

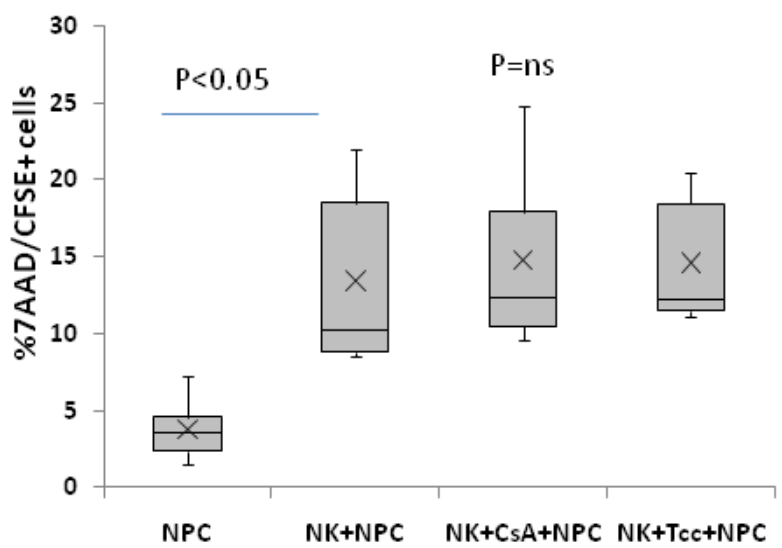

Figure 5: Cytotoxiciy of T cells and NK cells against NPC. A: Percentage of 7AAD/CFSE+ cells in presence of CD8 T cells expression of CD107 with or without cyclosporine A or tacrolimus. B: Percentage of 7AAD/CFSE+ cells in presence of NK cells expression of CD107 with or without cyclosporine A or tacrolimus. 
Citation: de Rham C, Tieng V, Tournier BB, Avila Y, Ginovart N, et al. (2013) The Role of Immunosuppression in the Transplantation of Allogenic Neural Precursors Derived from Human Pluripotent Stem Cells for Parkinson's Disease. J Stem Cell Res Ther S6: 006. doi:10.4172/21577633.S6-006

Page 7 of 9

CD107a expression by T-cells (Figure 6A) but not the one expressed by NK cells (Figure 6B).

\section{In vivo effect of immunosuppressive drugs}

To evaluate the importance of immunosuppressive and antiinflammatory treatments on survival and the stability of grafted cells, human embryonic stem cells (hESC) differentiated in neuroprecursors were transplanted into the striatum of adult male Sprague Dawley rats with different immunosuppressive regimen that included tacrolimus, cyclosporine and steroids. One-month post-implantation, the grafted cells survival was detected using the human cytoplasmic marker (HCM-ir) and the rat microglial cells response to implantation was observed using Iba1-ir. Without treatment, a few HCM-ir cells survive and numerous Ibal-ir amiboïd cells in the remaining graft suggest poor human neuroprecursor cells survival and strong rat microglial activation (Figures 7A-7C). In contrast, in rats treated with Tacrolimus, the survival of HCM-ir cells was better and the graft density of amiboïd Iba1-ir positive cells was decreased (Figure 7D-7F). Finally, in rats that received both immunosuppressive drugs (tacrolimus or cyclosporine) and anti-inflammatory treatments (prednisolone-Solumedrol), the survival rate of grafted human NPC was increased and the microglial density was low, and similar inside and outside the graft and of stellar morphology (Figures 7G-7L). These data suggest that both tacrolimus and cyclosporine, in association with an anti-inflammatory treatment, promote graft survival and minimise the host microglial response.

\section{Discussion}

Our data demonstrate the importance to evaluate ISD in the context of transplantation of pluripotent stem cells and their derivatives coming from genetically unrelated individuals. Several different ISD were used, and interestingly, whereas cyclosporine and tacrolimus possess the same final target, their effect in the maturation process is different in in vitro experiments but not in vivo. The inhibition of NPC differentiation by IvIG was expected, even though the IvIG was composed of a large variety of IgG coming from the pool of thousands of donors. The binding of IgG to any target like receptors, ligands... expressed by NPC would block critical signalling which activates the differentiation pathway. Mycophenolate mofetil (MMF) blocks selectively and reversibly the enzyme inosine monophosphate deshydrogenase which is critical for the synthesis of purine nucleotides and DNA replication. By its mode of action, MMF could interfere with neuron precursor division.

We focused our interest on the calcineurin inhibitor cyclosporine and tacrolimus because most of the published data on allogenic transplantation of pluripotent stem cells in in vivo models were performed with cyclosporine, mainly for historical reason. Although cyclosporine was the first calcineurin inhibitor that revolutionized immunosuppression by strongly reducing the rate of rejection in solid organ transplantation and still remains very effective, it is currently supplanted by tacrolimus. Previous data have suggested that cyclosporine could be deleterious in the maturation process of NPC [6,7], when other publications suggest that cyclosprorine could be beneficial $[8,9]$. Most of the published data have concluded that cycloporine had direct effect on neuron differentiation, but protected the engrafted cells from rejection $[8,9]$. Only data from Hunt et al. indicated that cyclosporine could have a direct positive on the maturation of adult neuron cells [10]. Direct and indirect effect can both play a positive role with regard to direct effect of NPC differentiation. While our previous in vitro data suggest that cyclosporine inhibits the maturation of NPC into mature neuron, the in vivo experiments with rats did not. Indeed, we did not observe significant differences after the transplantation of neuron precursors between rats treated with cyclosporine or tacrolimus (Figure 7). This can be due to differences in the levels of drugs between NPC directly exposed to cyclosporine in the culture dishes and transplanted NPC into the brain of rats that received the drugs by intraperitoneal injections. The state of neuron differentiation at the time of transplantation could be critical. The in vitro and in vivo experiments were not performed at the same stage of differentiation and we postulate that exposition of NPC to cyclosporine at early stage, as we did in the previous study, could be more deleterious than at a later stage as it was performed in vitro. Other parameters could also be important, like the doses, the site of administration per os, ip, or iv and the frequency of delivery. Jensen et al. [11] studied the survival of human NPC transplanted into rats in different conditions: cyclosporine given orally or injected was compared to no drug. Interestingly, the best result was a compromise of injections of cyclosporine followed by addition of cyclosporine in the drinking water. Our data demonstrated that NPC exposed in vitro to tacrolimus
A.

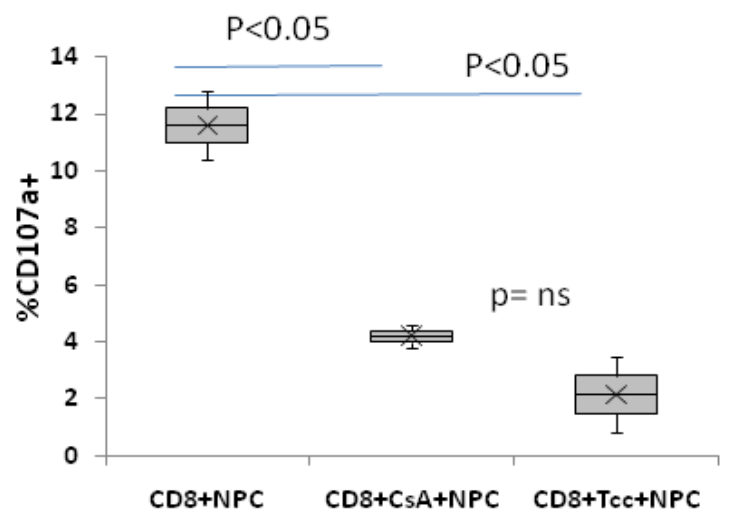

B.

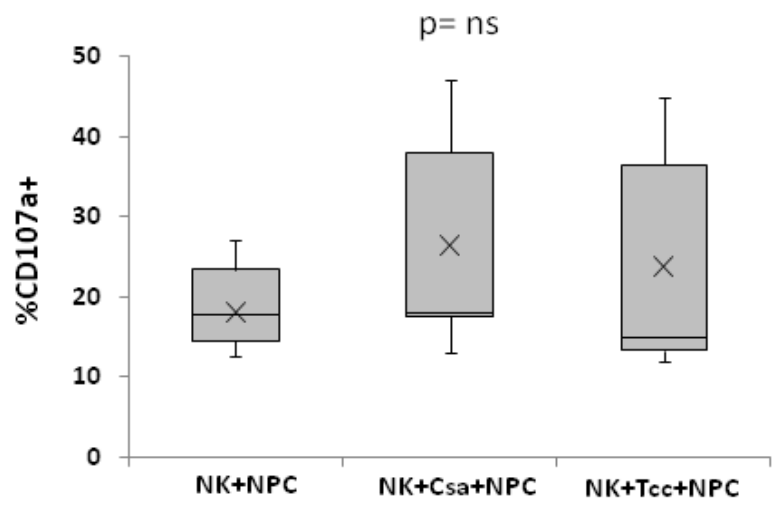

Figure 6: NK and T cell expression of CD107. A: CD8 T cells expression of CD107 is reduced in presence of CsA (cyclosporine A) or Tcc (tacrolimus). B: NK cells expression of CD107 is not influenced by the presence of CsA (cyclosporine A) or Tcc (tacrolimus). 
Citation: de Rham C, Tieng V, Tournier BB, Avila Y, Ginovart N, et al. (2013) The Role of Immunosuppression in the Transplantation of Allogenic Neural Precursors Derived from Human Pluripotent Stem Cells for Parkinson's Disease. J Stem Cell Res Ther S6: 006. doi:10.4172/21577633.S6-006

Page 8 of 9
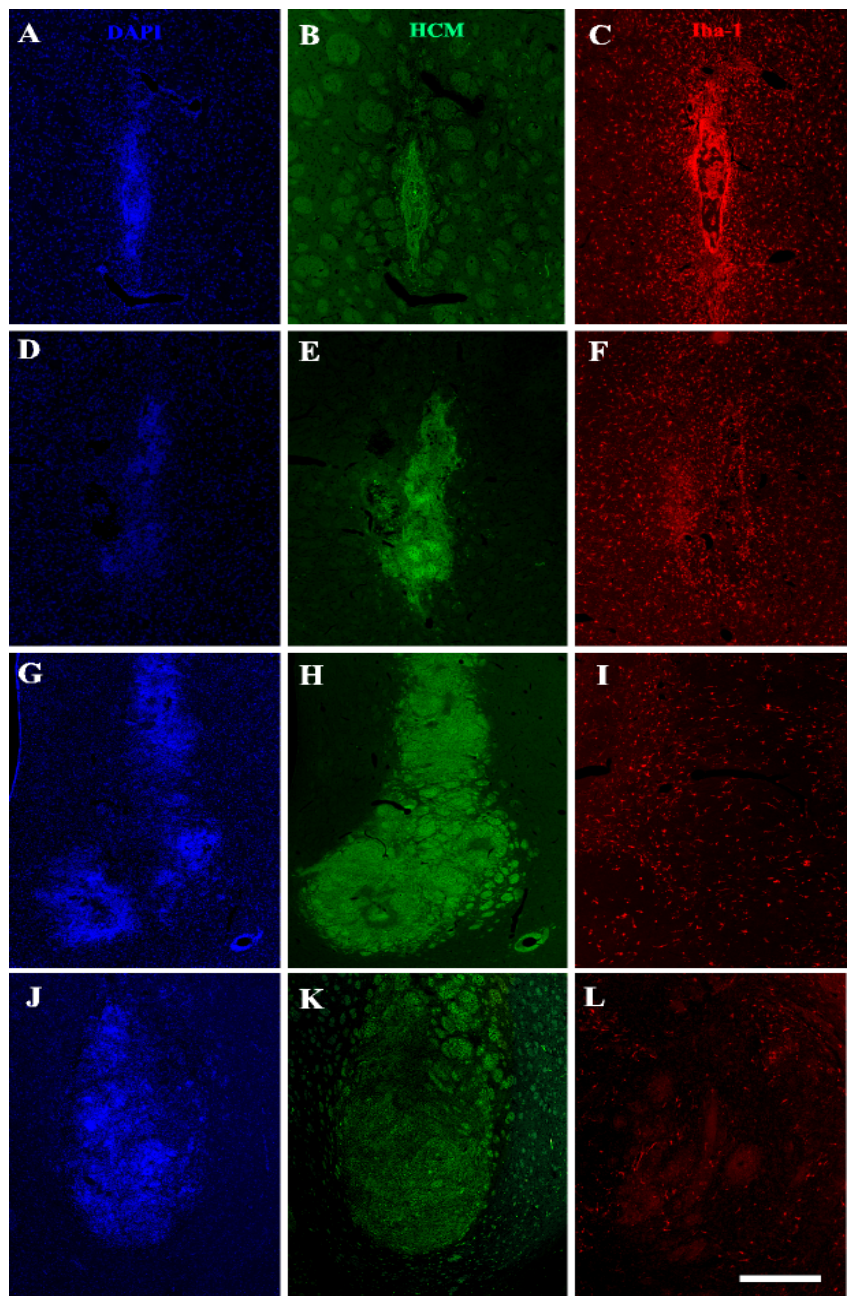

Figure 7: Immunohistochemistry of grafts in the rat striatum. Double immunohistochemical detection of grafted human cells (HCM-immunoreactivity in green) and of microglial cell detected (Iba1-immunoreactivity in red) in the striatum of implanted rats. Cell nuclei stained with DAPI (in blue). Following the implantation, rats were treated with saline 7A-7C, with tacrolimus only 7D-7F with tacrolimus and prednisolone 7G-7I or with cyclosporine and prednisolone 7J-7L. Scale bar: A, B, D, E, G, H, J ,K=600 $\mu \mathrm{m}, \mathrm{C}, \mathrm{F}, \mathrm{I}, \mathrm{L}=300 \mu \mathrm{m}$.

at early stage was not deleterious. The difference in the gene expression profile when NPC were differentiated in the presence of tacrolimus (or PBS) and cyclosporine indicated that the number of genes significantly modified by the two conditions ( $>$ or $<1.5$ fold increase) was not so important. Among them, the reduction of APOA-IV expression (6 fold decrease) is of interest. Previous works have demonstrated the importance of LDL and LDL receptor in the maturation of neurons $[12,13]$. In addition APOA-IV KO mice were associated with an acceleration of Alzheimer disease [14]. Further investigations are necessary to differentiate between a direct involvement of APOA-IV on neurogenesis and a lack of protective effect with regards to the toxicity of inflammation, oxidative stress mediated by APOA-IV on NPC.

Our results were generated with a specific protocol for NPC differentiation, and it remains to be demonstrated that similar results could be extrapolated to other experimental procedures of maturation of precursor cells. However, we believe that it could be important to evaluate the possible interference of the different ISD during the maturation process of any type of precursors and during transplantation. But in vitro experiment is not sufficient and should be confirmed by experimental model. In the rat model of NPC transplantation, the addition of prednisone to cyclosporine and tacrolimus for a few days after transplantation was clearly beneficial. Prednisone could have deleterious effect on neuron differentiation [6] but this problem is overcomed by the reduction of inflammation generated by the transplantation procedure. Inflammation has a direct harmful effect on the graft and activates the immune system to generate an adaptative immune response responsible of the rejection of the graft. Inflammation has already been shown to be suppressed by the differenciation of embryonic cell-derived neural precursor [15].

It was previously thought that hES cells possess immune-privileged properties, meaning that the immune cells would not kill hES cells. One reason for that is the low level of the major histocompatibility complex of class I (MHC-I) expression by pluripotent stem cells. First, our results indicated that CD8 T-cells were able to kill NPC suggesting that even a low level of MHC class-I is sufficient to be recognized by CD8 T-cells. Second, a low level of MHC class I on the cell surface of ES cells is a clear disadvantage with regard to the killing activity of NK cells which is linked to the absence or the low expression of MHC-I, ligand of the inhibitory KIR (killer Ig-like receptors) of NK cells. Although cyclosporine and tacrolimus are both able to block CD8 T-cells cytotoxicity, it is difficult to inhibit activated NK cells by classical ISD and it could represent hurdles for successful pluripotent stem cell transplantation. Several publications have already demonstrated the absence of significant effect of ISD on NK cells at least in vitro. Therefore, there is a need to find therapeutic agents that are able to block the killing activity of activated NK cells.

To prevent the rejection and the interference of ISD, the use of autologous human induced pluripotent stem cells (hiPS) seems to be the logical and the best solution. However, in 2011, Zhao et al. [16] published surprising results. The immunogenicity of mouse ES cells and mouse iPS cells was compared. Autologous ES cells were well tolerated without immunosuppression, whereas an immune rejection of autologous iPS through a specific T-cell response was detected. More recently, two publications in rodents suggest that iPS induce a very weak immune response, although the immunogenicity of iPS is heteregenous. In a non-human primate model, recent data established that autologous transplantation of iPSC-derived neurons elicited only a minimal immune response in the brain, in contrast to allograft which induce microglia activation and leucocytes infiltration [17]. The use of ISD must not be neglected, as long as such studies have not been done in different larger animal models, including human cells $[18,19]$.

Pluripotent stem cells like hES or iPS are certainly less immunogenic than adult somatic cells and interestingly, the two recent studies $[18,19]$ could suggest that pluripotent stem cells possess their own defense system. It has been already demonstrated that hES cells adopt at least two mechanisms to escape lysis by NK and T-cells. One is based on CD95L and FasL, which induce the killing of cells expressing CD95 and Fas, and the second involves the high expression of SPI-9, a serine protease inhibitor $[20,21]$. Because iPS are generated form different somatic cells with different protocols, immunogenicity of distinct iPS cell line and their derived tissues could be different and should be evaluated in each cases [22,23].

From a practical point of view, the possibility of generating autologous iPS for each recipient seems to be a difficult or an impossible task for several reasons, like regulation processes, correction of genetic diseases or genetic instability. Differences in the methods used for generation and culture of hiPS cell lines is a critical point 
Citation: de Rham C, Tieng V, Tournier BB, Avila Y, Ginovart N, et al. (2013) The Role of Immunosuppression in the Transplantation of Allogenic Neural Precursors Derived from Human Pluripotent Stem Cells for Parkinson's Disease. J Stem Cell Res Ther S6: 006. doi:10.4172/21577633.S6-006

Page 9 of 9

and the question of establishing a hiPS bank with well defined genetic characteristics is on debate. These hiPS would cover a large panel of population, so each individual would be matched with a specific hiPS cell line $[23,24]$. Such cell lines could also be tested in presence of ISD to validate the absence of interference on cell differentiation steps as well as their impact on maturation and cell functionality.

Currently most of the data have been performed in animal models, mainly rodents but also large animals such as pigs or nonhuman primates. Each type of animal has its own advantages and disadvantages. An important aspect of animal studies is the possibility to test immune responses to pluripotent stem cells from different origins like embryonic stem cells or induced pluripotent stem cells and their derivatives, and also the possibility to test the best ISD regimen which is mandatory to prevent rejection. Small animals, such as mice or rats, are important models for immunological discoveries and serve as proof of concepts, whereas larger animals are important, as their immunophysiology is closer to humans comparing to smaller animals [25].

\section{Conclusion}

Our data indicate that immunosuppression is mandatory to prevent the rejection of pluripotent stem cells or their derivative, like neurons. In vitro analysis cannot always predict the efficacy of immunossuppression used in vivo. The addition of anti-inflammatory drugs like prednisolone seems to be important to increase the graft survival. Natural killer cells are not inhibited by classical immunosuppressive drugs and could be harmful for the transplanted cells or tissue.

\section{Competing Interests}

The authors declare that they have no competing interests

\section{Acknowledgment}

The authors would like to acknowledge support through the FP7-funded ESNATS program (Grant Agreement number: FP7 - 201619). This project was supported by the Swiss national foundation grant N ${ }^{\circ} \mathrm{CRSI33-125408}$ (Synergia grant), by the Fonds Gustaaf Hamburger of the Philanthropia foundation and by the Swiss Parkinson.

\section{References}

1. Drukker M, Katchman H, Katz G, Even-Tov Friedman S, Shezen E, et al (2006) Human embryonic stem cells and their differentiated derivatives are less susceptible to immune rejection than adult cells. Stem Cells 24: 221-229.

2. Li L, Baroja ML, Majumdar A, Chadwick K, Rouleau A, et al. (2004) Human embryonic stem cells possess immune-privileged properties. Stem Cells 22: 448-456.

3. Robertson NJ, Brook FA, Gardner RL, Cobbold SP, Waldmann H, et al. (2007) Embryonic stem cell-derived tissues are immunogenic but their inherent immune privilege promotes the induction of tolerance. Proc Natl Acad Sci U S A 104: 20920-20925.

4. Ferrari-Lacraz S, Aubert V, Buhler L, Pascual M, Andresen I, et al. (2006) AntiHLA antibody repertoire after IVIg infusion in highly sensitized patients waiting for kidney transplantation. Swiss Med Wkly 136: 696-702.

5. Jordan SC, Tyan D, Stablein D, McIntosh M, Rose S, et al. (2004) Evaluation of intravenous immunoglobulin as an agent to lower allosensitization and improve transplantation in highly sensitized adult patients with end-stage renal disease: report of the NIH IG02 trial. J Am Soc Nephrol 15: 3256-3262.

6. Preynat-Seauve O, de Rham C, Tirefort D, Ferrari-Lacraz S, Krause $\mathrm{KH}$, et al. (2009) Neural progenitors derived from human embryonic stem cells are targeted by allogeneic T and natural killer cells. J Cell Mol Med 13: 3556-3569.

7. Schwarz SC, Wittlinger J, Schober R, Storch A, Schwarz J (2006) Transplantation of human neural precursor cells in the 6-OHDA lesioned rats: effect of immunosuppression with cyclosporine A. Parkinsonism Relat Disord 12: $302-308$.

8. Funk WD, Labat I, Sampathkumar J, Gourraud PA, Oksenberg JR, et al.
(2012) Evaluating the genomic and sequence integrity of human ES cell lines; comparison to normal genomes. Stem Cell Res 8: 154-64.

9. Lu HZ, Wang YX, Zhou JS, Wang FC, Hu JG (2010) Cyclosporin A increases recovery after spinal cord injury but does not improve myelination by oligodendrocyte progenitor cell transplantation. BMC Neurosci 11: 127.

10. Hunt J, Cheng A, Hoyles A, Jervis E, Morshead CM (2010) Cyclosporin A has direct effects on adult neural precursor cells. J Neurosci 30: 2888-2896.

11. Jensen M, Krishnaney-Davison R, Cohen LK, Zhang SC (2012) Injected versus oral cyclosporine for human neural progenitor grafting in rats. J Stem Cell Res Ther.

12. Mulder M, Jansen PJ, Janssen BJ, van de Berg WD, van der Boom $\mathrm{H}$, et al (2004) Low-density lipoprotein receptor-knockout mice display impaired spatia memory associated with a decreased synaptic density in the hippocampus. Neurobiol Dis 16: 212-219.

13. Parada C, Escola-Gil JC, Bueno D (2008) Low-density lipoproteins from embryonic cerebrospinal fluid are required for neural differentiation. J Neurosc Res 86: 2674-2684.

14. Cui Y, Huang M, He Y, Zhang S, Luo Y (2011) Genetic ablation of apolipoprotein A-IV accelerates Alzheimer's disease pathogenesis in a mouse model. Am J Pathol 178: 1298-1308.

15. Ideguchi M, Shinoyama M, Gomi M, Hayashi H, Hashimoto N, et al. (2008) Immune or inflammatory response by the host brain suppresses neuronal differentiation of transplanted ES cell-derived neural precursor cells. J Neurosci Res 86: 1936-1943.

16. Zhao T, Zhang ZN, Rong Z, Xu Y (2011) Immunogenicity of induced pluripotent stem cells. Nature 474: 212-215.

17. Morizane A, Doi D, Kikuchi T, Okita K, Hotta A, et al. (2013) Direct Comparison of Autologous and Allogeneic Transplantation of iPSC-Derived Neural Cells in the Brain of a Nonhuman Primate. Stem Cell Reports 1: 283-292.

18. Araki R, Uda M, Hoki Y, Sunayama M, Nakamura M, et al. (2013) Negligible immunogenicity of terminally differentiated cells derived from induced pluripotent or embryonic stem cells. Nature 494: 100-104

19. Guha P, Morgan JW, Mostoslavsky G, Rodrigues NP, Boyd AS (2013) Lack of immune response to differentiated cells derived from syngeneic induced pluripotent stem cells. Cell Stem Cell 12: 407-412.

20. Medema JP, de Jong J, Peltenburg LT, Verdegaal EM, Gorter A, et al. (2001) Blockade of the granzyme B/perforin pathway through overexpression of the serine protease inhibitor PI-9/SPI-6 constitutes a mechanism for immune escape by tumors. Proc Natl Acad Sci U S A 98: 11515-11520.

21. Utermohlen O, Kronke M (2007) Survival of priceless cells: active and passive protection of embryonic stem cells against immune destruction. Arch Biochem Biophys 462: 273-277.

22. Di Trapani M, Bassi G, Ricciardi M, Fontana E, Bifari F, et al. (2013) Comparative Study of Immune Regulatory Properties of Stem Cells Derived from Different Tissues. Stem Cells Dev 22: 2990-3002.

23. Kaneko S, Yamanaka S (2013) To be immunogenic, or not to be: that's the iPSC question. Cell Stem Cell 12: 385-386.

24. Zimmermann A, Preynat-Seauve O, Tiercy JM, Krause KH, Villard J (2012) Haplotype-based banking of human pluripotent stem cells for transplantation: potential and limitations. Stem Cells Dev 21: 2364-2373.

25. Cibelli J, Emborg ME, Prockop DJ, Roberts M, Schatten G, et al. (2013) Strategies for improving animal models for regenerative medicine. Cell Stem Cell 12: $271-274$

This article was originally published in a special issue, Transplantation Immunology handled by Editor(s). Qing Ma, University of Texas, USA 